\title{
Experimental and numerical acoustic characterization of ultrasonically absorptive porous materials
}

\author{
Alexander Wagner* and Jan Martinez Schramm ${ }^{\dagger}$ \\ German Aerospace Center (DLR), Institute of Aerodynamics and Flow Technology, Göttingen, Germany \\ Christian Dittert ${ }^{*}$ \\ German Aerospace Center (DLR), Institute of Structures and Design, Stuttgart, Germany
}

Victor C. B. Sousa ${ }^{\S}$, Danish Patel ${ }^{\mathrm{II}}$ and Carlo Scalo"

School of Mechanical Engineering, Purdue University, West Lafayette, USA

\begin{abstract}
The paper addresses the experimental and numerical acoustic characterization of ultrasonically absorptive porous materials with random microstructure such as carbon fiber reinforced carbon ceramic $\mathrm{C} / \mathrm{C}$ or $\mathrm{C} / \mathrm{C}-\mathrm{SiC}$. The present study carries on a preceding experimental study of the authors by improving and extending the established experimental method and by conducting complementary numerical studies. The latter uses a new inverse Helmholtz Solver associated with an incident planar acoustic wave to estimate the complex acoustic impedance at the open surface of an arbitrarily shaped cavity. The experimental approach is complemented by high speed schlieren visualization and Mach-Zehnder Interferometer measurements to qualitatively and quantitatively assess the interaction between an ultrasonic wave packet and a porous surface. The main emphasis of the paper is to assess the potential and the limitations of the experimental methods and the comparison of the experimental results to the numerically obtained absorption characteristics.
\end{abstract}

\section{Introduction}

In flow fields with small free-stream disturbances, negligible surface roughness and predominantly two-dimensional hypersonic boundary layers second-mode instabilities are known to be the dominant mechanism leading to transition. Due to the acoustic nature of the instability ultrasonically absorptive coatings have the potential to damp the instabilities and thus delay laminar-turbulent transition [1]. Preceding studies in hypersonic wind tunnels indeed revealed that porous surfaces, such as metal sheets with cylindrical blind holes, felt metal and carbon fiber reinforced carbon ceramic (C/C) can be used to damp hypersonic boundary layer instabilities and thus to passively control boundary layer transition. [2, 9 ]

However, to achieve practical relevance the ultrasonically absorptive materials need to withstand the high shear stresses and heat loads present in hypersonic sustained flight. Such a material is for instance C/C-SiC which has been used successfully as TPS on hypersonic vehicles, [10, 11], and which can be manufactured with a comparable porosity and microstructure as found on $\mathrm{C} / \mathrm{C}$.[12] To assess the potential of such a material with respect to transition control the requirement arises to determine the ultrasonic absorption characteristic at the surface. For this purpose a test rig was set up at DLR Göttingen to measure the reflection coefficient at varying static pressures as reported in Wagner et al. [13]. The improved setup uses ultrasonic sound transducers covering a frequency range of $125 \mathrm{kHz}$ to $490 \mathrm{kHz}$ and thus include a wide range of the frequency domain of interest with respect to the second mode instabilities.

On the numerical side, the inverse Helmholtz solver methodology [14] is used to directly evaluate the broadband acoustic impedance associated with each surface cavity of the material, which are then combined to quantify the overall acoustic absorption characteristics of the sample considered. A brief discussion of the iHS methodology and its context in the current study is provided in section $B$. A detailed description and validation of the methodology can be found in Patel et al. [15].

\footnotetext{
*Research Scientist, Spacecraft Department, Alexander.Wagner@ dlr.de

$\dagger$ Group Leader Aerothermodynamics, Spacecraft Department, Jan.Martinez@dlr.de

${ }^{\ddagger}$ Research Scientist, Department of Space System Integration, Christian.Dittert@dlr.de

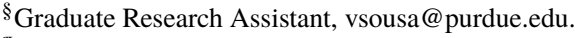

"I Graduate Research Assistant, AIAA Student Member, pate1472@purdue.edu

॥ Assistant Professor, AIAA Member, scalo@purdue.edu
} 


\section{Method}

\section{A. Experimental Setup}

Ultrasonic air-coupled PZT ${ }^{\text {f }}$ sound transducers with specific resonance frequencies are arranged pairwise to transmit ultrasonic wave packets towards a material of interest and to record the wave packet after its reflection at the surface. Sound transducers with resonance frequencies of $125 \mathrm{kHz}, 225 \mathrm{kHz}, 290 \mathrm{kHz}, 300 \mathrm{kHz}, 405 \mathrm{kHz}$ and $490 \mathrm{kHz}$ are installed at an angle of incidence of $30^{\circ}$ and a distance of $40 \mathrm{~mm}$ such that the transducer axes intersect at the sample surface. Figure 1 shows a schematic of the setup while table 1 provides the geometrical parameters introduces in the figure. The angle of incidence was chosen based on experimental work performed by Fedorov et al. [16]. Further, it is

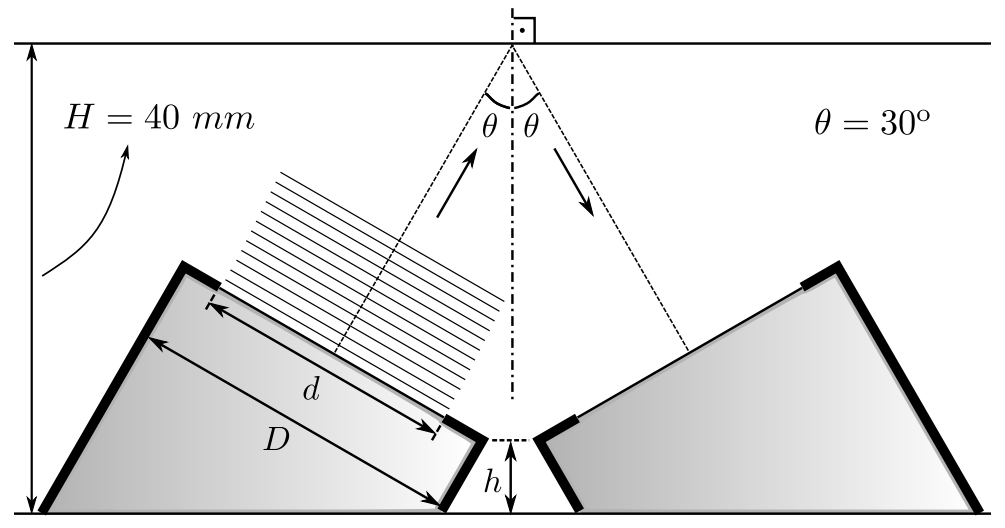

Fig. 1 Schematic of the experimental setup using ultrasonic sound transducers (not to scale).

justified by a numerical investigation conducted by Brès et al. [17] showing that in all cases relevant for ultrasonically absorptive coatings the angle of incidence of second mode waves is smaller than $26^{\circ}$. Further information on the experimental setup of the sound transducers is provided in Wagner et al. [13].

\begin{tabular}{c|c|c|c}
$f[\mathrm{kHz}]$ & $d[\mathrm{~mm}]$ & $D[\mathrm{~mm}]$ & $h[\mathrm{~mm}]$ \\
\hline 125 & 19 & 30 & 1 \\
223 & 11 & 17 & 0 \\
289 & 7 & 17 & 0.7 \\
300 & 7 & 17 & 1 \\
405 & 9 & 17 & 0 \\
490 & 8 & 17 & 0
\end{tabular}

Table 1 Geometrical parameters of the transducer/receiver pairs.

A high speed Schlieren setup (in a z-type shaped arrangement) is used to visualize the generated sound waves as they propagate towards the test sample at which they are reflected. The optical setup is illuminated by a Cavitar Cavilux Smart laser system, which is operated with a pulse width of $20 \mathrm{~ns}$. A Phantom v2012 camera is used with a resolution of $640 \times 480 \mathrm{px}^{2}$ at a frame rate of $70 \mathrm{kHz}$ to capture the development of the sound waves. No other light than that from the laser system exposes the camera chip, which means that the pulse width of the laser determines the exposure time. Quantitative measurements beyond the Schlieren visualisation are planned using an interferometric method, which is based on the interference pattern formed by the superposition of light waves which originate from the same coherent source (in this case the Cavitar Cavilux Smart laser system) but traverse different paths. The fringe pattern formed by these beams indicates the local phase shifts arising from the difference in the optical paths traversed by the interfering beams, which will be in our case a reference cell and the test rig. The most common interferometers are Michelson and

*Plumb-Zirconate-Titanate piezoelectric ceramic 
Mach-Zehnder ones; we plan to used the latter one. The sensitivity of the existing Mach-Zehnder and the mathematical processing of the interference pattern data allows us to measure the density formed by the acoustic waves in the gas. Relying in the assumption that we will expect pressure fluctuations in the range of $20 \mathrm{~Pa}$ up to $70 \mathrm{~Pa}$, leading to a density change of minimal $0.2 \mathrm{~g} / \mathrm{m}^{3}$ at room temperature and test gas air, we expect a minimum phase shift order of 3.5 , which should give us a reasonable experimental uncertainty in the density measurement.

\section{B. Numerical Setup}

A novel methodology to determine the spatial distribution of the complex acoustic impedance at the open surface of an arbitrarily shaped geometry, associated with an incident planar acoustic wave of assigned wavenumber vector and frequency was developed in the first inverse Helmholtz Solver (iHS) [14, 15]. This code reconstructs, at a given
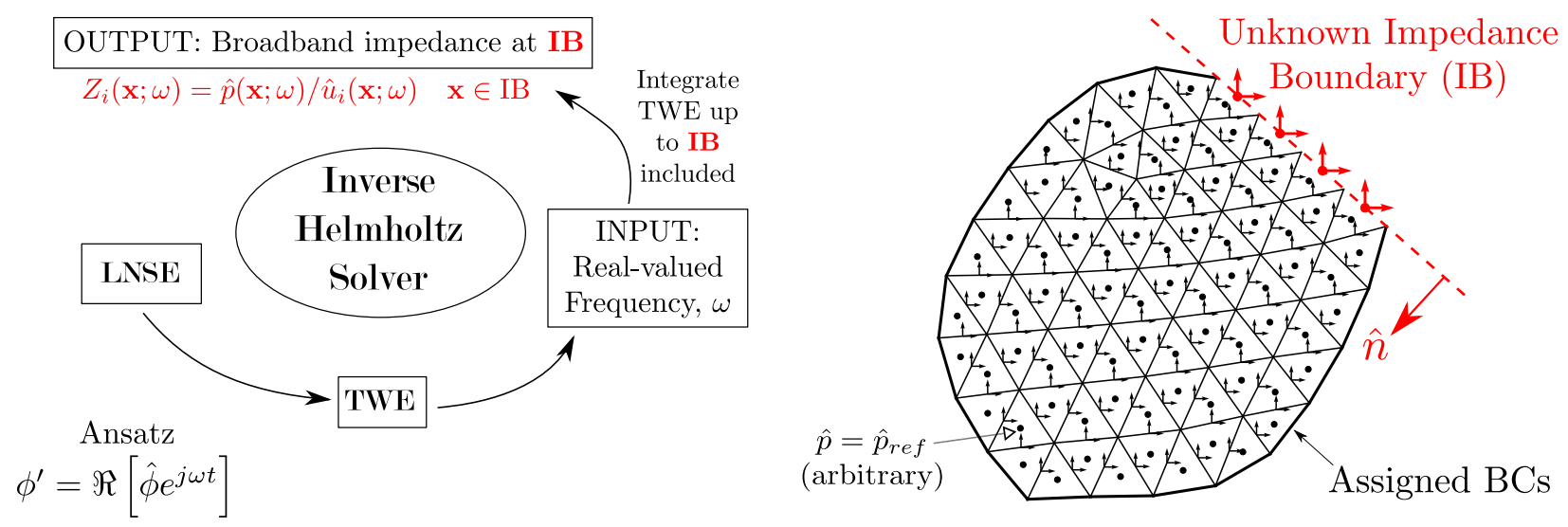

Fig. 2 Inverse Helmholtz solver procedure used to calculate the impedance of cavities typical of blind-hole porous surfaces.

frequency, the complex acoustic waveform in the cavity up to the open surface or unknown impedance boundary (IB), hereby providing the complete spatial distribution of impedance as a result of the calculation. Multiple instances of the iHS can be executed in parallel for different frequencies, allowing for a rapid determination of the full broadband impedance at every point of the IB. From a high definition image of the $\mathrm{C} / \mathrm{C}$ block (figure 3), large cavity edges are
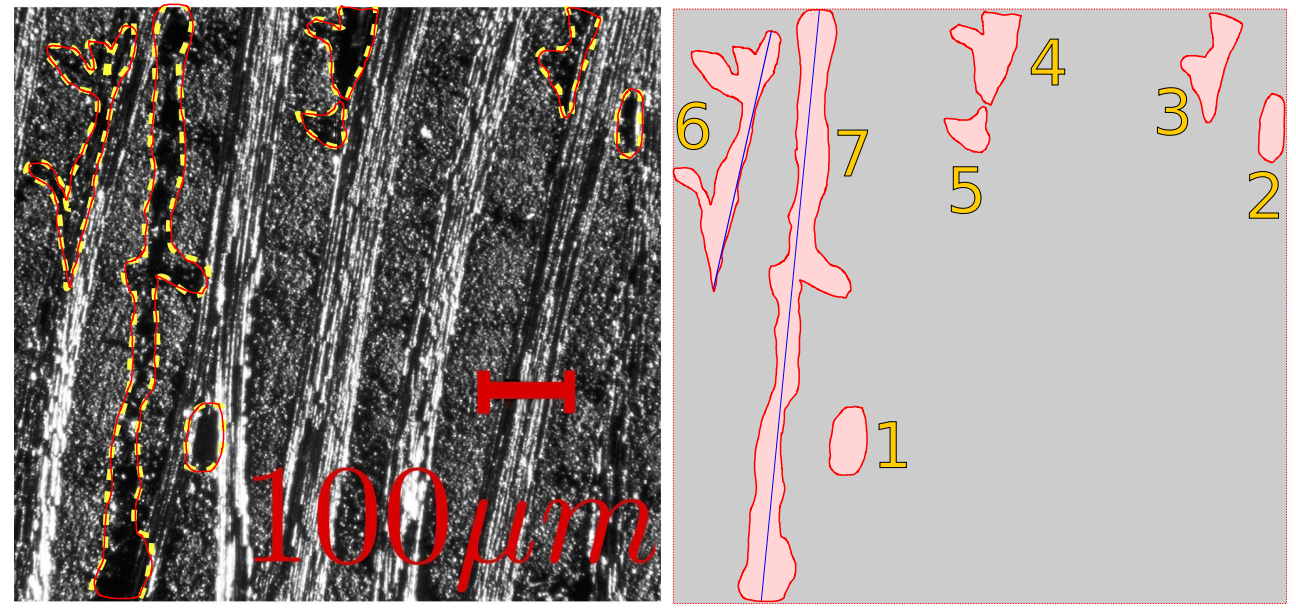

Fig. 3 Inverse Helmholtz solver procedure used to calculate the impedance of cavities typical of blind-hole porous surfaces.

detected and meshed to be fed as input to the iHS. The impedance for various cavities are then evaluated by the solver and combined together-noting that the admittance of the surrounding hard surface is zero-to yield the effective, surface averaged impedance of the block. 


\section{Preliminary Results}

\section{A. Experimental}

The high speed schlieren setup described in section A was used to visualize the ultrasonic waves generated by the ultrasonic sound transducers in the the above introduced setup. Although the signal to noise ration is low, waves at $125 \mathrm{kHz}, 300 \mathrm{kHz}$ and $490 \mathrm{kHz}$ were successfully visualized at ambient pressures as shown in figure 4 . The schlieren images resemble the schematic in figure 1 in which the ultrasonic wave packets are generated by the transducer on the left. Subsequently the waves propagate along the transducer axis towards the investigates sample surface at the top of the image and are reflected back towards the second transducer. The schlieren visualizations provide vital qualitative information for the numerical part of the study.

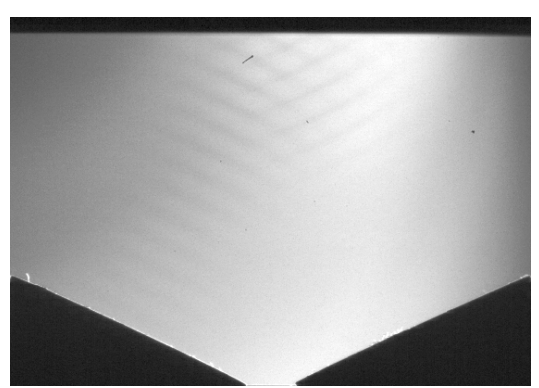

(a) $125 \mathrm{kHz}$

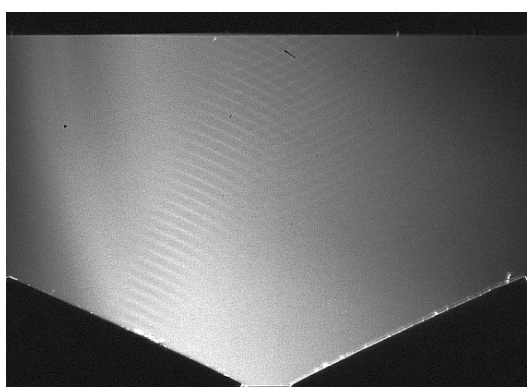

(b) $300 \mathrm{kHz}$

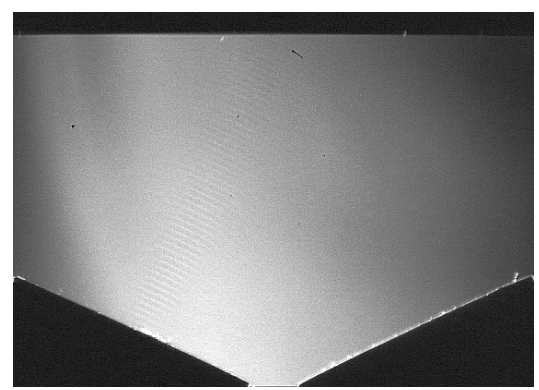

(c) $490 \mathrm{kHz}$

Fig. 4 Schlieren visualizations of ultrasonic waves at $125 \mathrm{kHz}, 300 \mathrm{kHz}$ and $490 \mathrm{kHz}$ at ambient pressure.

Figure 5 provides first results on the measured absorption coefficient of a $\mathrm{C} / \mathrm{C}$ sample using the optimized experimental setup. The absorption coefficient is provided as function of pressure and a frequency range of $125 \mathrm{kHz} \mathrm{zu}$ $490 \mathrm{kHz}$.

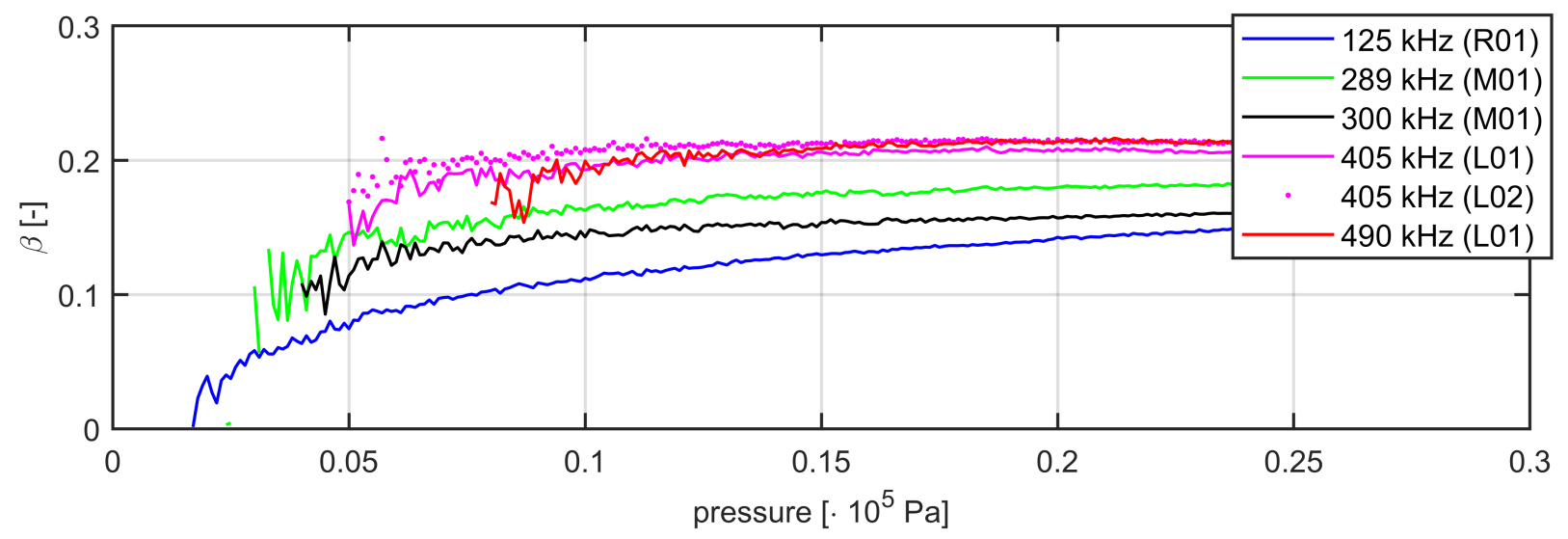

Fig. 5 Absorption coefficient experimentally obtain on a C/C sample.

\section{B. Numerical}

The iHS was used to evaluate the effective surface impedance of the carbon-carbon ultrasonically absorptive coating (C/C UAC) at the experiment conditions and these results were rearranged into the reflection coefficient, a quantity that has more physical meaning to this analysis since it is the ratio of the reflected wave over the incoming wave. Since a solid surface reflection does not change the phase or amplitude of a wave, the comparison between the wave reflected of a solid and porous surface is directly related to the reflection coefficient. Figure 6 and 7 provide the first estimates of the 
real and imaginary components of the reflection coefficient as well as the phase angle of the reflection coefficient as function of frequency and static pressure.
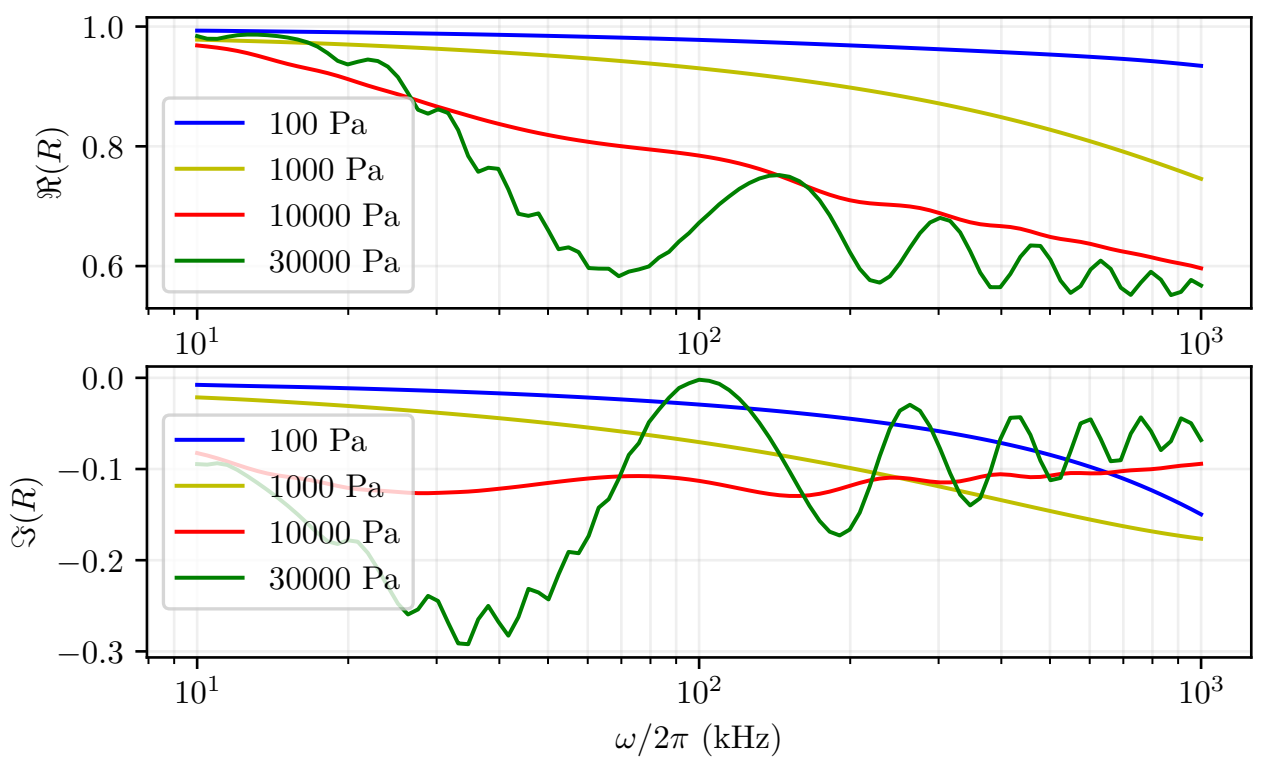

Fig. 6 Real and imaginary components of the reflection coefficient.

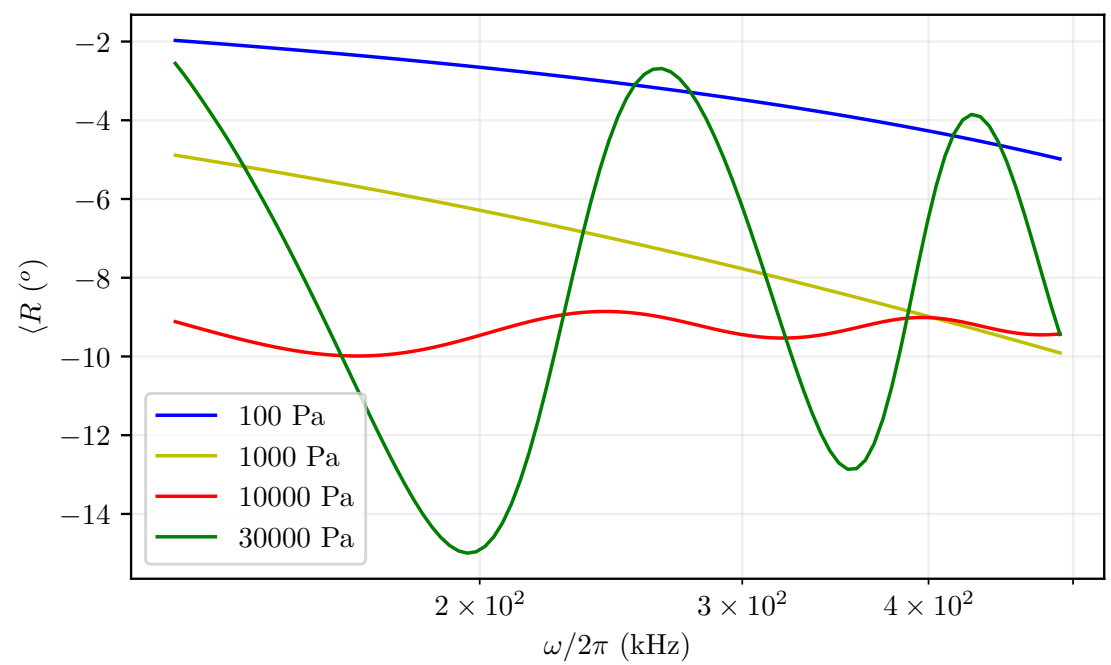

Fig. 7 Phase angle of the reflection coefficient.

\section{Acknowledgments}

The authors acknowledge the support of the Rosen Center for Advanced Computing (RCAC) at Purdue, the Air Force Office of Scientific Research (AFOSR) grant FA9550-16-1-0209 and grant FA9550-16-1-0456 and the very fruitful discussions with Dr. Ivett Leyva (AFOSR). 


\section{References}

[1] Malmuth, N., Fedorov, A., Shalaev, V., Cole, J., Khokhlov, A., Hites, M., and Williams, D., "Problems in High Speed Flow Prediction Relevant to Control," 2nd AIAA, Theoretical Fluid Mechanics Meeting, 1998. doi:10.2514/6.1998-2695, aIAA 98-2695.

[2] Fedorov, A. V., Malmuth, N. D., Rasheed, A., and Hornung, H. G., "Stabilization of Hypersonic Boundary Layers by Porous Coatings," AIAA, Vol. 39, No. 4, 2001, pp. 605-610.

[3] Rasheed, A., Hornung, H. G., Fedorov, A. V., and Malmuth, N. D., "Experiments on Passive Hypervelocity Boundary-Layer Control Using an Ultrasonically Absorptive Surface,” AIAA, Vol. 40, No. 3, 2002, pp. 481-489.

[4] Fedorov, A. V., Kozlov, V., Shiplyuk, A., Maslov, A., and Malmuth, N., "Stability of Hypersonic Boundary Layer on Porous Wall with Regular Microstructure,” AIAA Journal, Vol. 44, No. 8, 2006, pp. 1866-1871.

[5] Fedorov, A. V., Shiplyuk, A., Maslov, A., Burov, E., and Malmuth, N., "Stabilization of a hypersonic boundary layer using an ultrasonically absorptive coating,” Journal of Fluid Mechanics, Vol. 479, 2003, pp. 99-124. doi:10.1017/S0022112002003440.

[6] Maslov, A. A., Shiplyuk, A., Sidorenko, A., Polivanov, P., Fedorov, A., Kozlov, V., and Malmuth, N., "Hypersonic Laminar Flow Control Using a Porous Coating of Random Microstructure," 44th AIAA Aerospace Sciences Meeting and Exhibit, Reno, Nevada, 2006. AIAA 2006-1112.

[7] Maslov, A. A., Fedorov, A. V., Bountin, D. A., Shiplyuk, A. N., Sidorenko, A. A., Malmuth, N., and Knauss, H., "Experimental study of transition in hypersonic boundary layer on ultrasonically absorptive coating with random porosity," 46th AIAA Aerospace Sciences Meeting and Exhibit, Reno, Nevada, 2008. AIAA 2008-587.

[8] Lukashevich, S. V., Morozov, S. O., and Shiplyuk, A. N., "Experimental study of the effect of a passive porous coating on disturbances in a hypersonic boundary layer 1. Effect of the porous coating length," Journal of Applied Mechanics and Technical Physics, Vol. 54, No. 4, 2013, pp. 572-577. doi:10.1134/S002189441304007X.

[9] Wagner, A., Hannemann, K., Wartemann, V., and Giese, T., "Hypersonic boundary-layer stabilization by means of ultrasonically absorptive carbon-carbon material - Part 1: Experimental Results," 51st AIAA Aerospace Sciences Meeting, Texas, 2013. doi:10.2514/6.2013-270, URL http://dx.doi.org/10.2514/6.2013-270 aIAA 2013-0270.

[10] Turner, J., Hörschgen, M., Jung, W., Stamminger, A., and Turner, P., "SHEFEX Hypersonic Re-entry Flight Experiment; Vehicle and Subsystem Design, Flight Performance and Prospects," 14th AIAA/AHI Space Planes and Hypersonic Systems and Technologies Conference, 2006. AIAA 2006-8115.

[11] Weihs, H., Longo, J., and Turner, J., "Key Experiments within the SHEFEX II Mission,” IAC 2008, Glasgow, Scottland UK, 2008.

[12] Wagner, A., Wartemann, V., Hannemann, K., Kuhn, M., and Dittert, C., "The Potential of Ultrasonically Absorptive TPS Materials for Hypersonic Vehicles," 20th AIAA International Space Planes and Hypersonic Systems and Technologies Conference, 2015.

[13] Wagner, A., Hannemann, K., and Kuhn, M., "Ultrasonic absorption characteristics of porous carbon-carbon ceramics with random microstructure for passive hypersonic boundary layer transition control," Experiments in Fluids, Vol. 55, No. 6, 2014, 1750. doi:10.1007/s00348-014-1750-4.

[14] Patel, D., Gupta, P., Scalo, C., Rothermel, T., and Kuhn, M., “Towards Impedance Characterization of Carbon-Carbon Ultrasonically Absorptive Coatings via the Inverse Helmholtz Problem," 55th AIAA Aerospace Sciences Meeting, 2017 , p. 0460. doi:10.2514/6.2017-0460, URL http://dx.doi .org/10.2514/6.2017-0460, aIAA-2017-0460.

[15] Patel, D., Gupta, P., and Scalo, C., "Impedance Calculation via the Inverse Helmholtz Solver (iHS)," arXiv preprint arXiv:1708.02069, 2017.

[16] Fedorov, A. V., Kozlov, V. F., and Addison, R. C., "Reflection of acoustic disturbances from a porous coating of regular microstructure," 5th AIAA Theoretical Fluid Mechanics Conference, 2008. AIAA 2008-3902.

[17] Brès, G. A., Colonius, T., and Fedorov, A. V., "Acoustic Properties of Porous Coatings for Hypersonic Boundary-Layer Control," AIAA Journal, Vol. 48, No. 2, 2010, pp. 267-274. doi:10.2514/1.40811. 